\title{
Il Teorema di Unicità nella Dinamica \\ dei Fluidi Compressibili
}

\section{DARIO GRAFFI}

Istituto Matematico "Salvatote Pincherle", Università di Bologna

1. Nel suo recente volume, dedicato all'idrodinamica, Garrett Birkhoff ${ }^{1}$ osserva che, per una rigorosa teoria dei modelli, è necessario appoggiarsi a un teorema di unicità per le equazioni del moto dei fluidi ${ }^{2}$. A proposito di questo teorema egli cita le ricerche, condotte con mezzi piuttosto elevati, di L. Lichtenstein (1927) e le ricerche di J. Leray (1934) e K. Maruhn (1939) che si riferiscono però al caso, troppo ideale per la teoria dei modelli, di un fluido che occupa tutto lo spazio. E bene però aggiungere che, fin dal 1929, E. Foà ${ }^{3}$ aveva indicato una dimostrazione, relativamente semplice, del teorema in discorso, valida forse sotto ipotesi più larghe di quelle di Lichtenstein; dimostrazione che fu da me perfezionata l'anno successivo ed estesa alle equazioni della propagazione del calore per convezione naturale. Però, in tutti i lavori finora citati si suppone il fluido incompressibile (o meglio nulla la divergenza della velocità) forse anche par tale ragione il Birkhoff ritiene finora sconosciuto il teorema di unicità per le equazioni della dinamica dei fluidi.

Nella presente Nota proveremo il suddetto teorema per le equazioni dei fluidi, compressibili, barotropici ${ }^{5}$, omogenei, cioè per le equazioni che il Birkhoff scrive nel suo libro. Naturalmente il nostro risultato verrà ottenuto in base ad opportune condizioni iniziali e al contorno, e ad alcune ipotesi di carattere fisico e di regolarità per le soluzioni delle equazioni in discorso; condizioni e ipotesi, a mio avviso, abbastanza ampie (per quanto sia desiderabile vengano migliorate), e che ora andremo ad esporre.

${ }^{1}$ G. Birkhoff-Hydrodynamics-Princeton University Press 1950 (pp. 96-97).

${ }^{2}$ Questa osservazione è stata fatta, in sostanza, fin dal 1928 da E. Fò̀ nella Nota "Sulle basi dell'analisi dimensionale"-L'industria XLII, 1928.

${ }^{3}$ E. Fò̀-"Sull'impiego dell'analisi dimensionale nello studio del moto turbolento"L'industria XLIII, 1929.

${ }^{4} \mathrm{D}$. Graffi-"Sulla teoria della propagazione del calore per convezione naturale"Rend. Lincei $\left(6^{a}\right)$ XII (1930) pp. 129-135.

${ }^{5}$ Come è noto, un fluido si dice barotropico se è assegnata una relazione fra la sua pressione e la sua densità. 
2. Le equazioni per il moto dei fluidi barotropici viscosi sono $^{6}$ :

$$
\begin{aligned}
\rho \frac{\partial \mathbf{v}}{\partial t}+\rho \frac{d \mathbf{v}}{d P} \mathbf{v} & =-\operatorname{grad} p+\mathrm{F}(\rho, P, t)+\operatorname{grad} \beta \\
\frac{\partial \rho}{\partial t}+\operatorname{div}(\rho \mathbf{v}) & =0 \\
p & =f(\rho)
\end{aligned}
$$

dove $\rho, \mathbf{v}, p$ indicano, rispettivamente, la densità, la velocità, la pressione in un punto $P$ del dominio $(D)$ in cui si muove il fluido, $\mathbf{F}(\rho, P, t) d V$ la forza agente, sull'elemento $d V$ del fluido, intorno al punto $P$ e all'istante $t$ (supporremo questa forza assegnata come funzione di $\rho, P$ e del tempo $t$ ). L'omografia $\beta$ rappresenta il tensore dovuto alla viscosità che puo scriversi:

$$
\beta=2 \mu D \frac{d \mathbf{v}}{d P}+\lambda \operatorname{div} \mathbf{v}
$$

dove $\mu$ e $\lambda$ indicano i coefficienti di viscosità del fluido ${ }^{7}$. La relazione (3) esprime appunto che il fluido è barotropico. Noi faremo poi sulle (1), (2), (3), (4) le seguenti ipotesi. La $f(\rho)$ e la sua derivata prima $f^{\prime}(\rho)$ siano funzioni continue e positive di $\rho$ per ogni $\rho>0$, inoltre $f^{\prime \prime}(\rho)$ continua per $\rho>0$; queste ipotesi sono certamente soddisfatte se, come si suppone in molti casi, $p=a \rho^{\gamma}$ con $a$ e $\gamma$ positive. Supporremo inoltre $\mu$ e $\lambda$ indipendenti da $\rho$ (il che avviene nei gas se la temperatura rimane costante), che nel fluido si abbia sempre energia dissipata per viscosità cioè $I_{1}(\beta D(d v / d P)) \geqq 0$ e infine la $\mathrm{F}(\rho, P, t)$ funzione continua e limitata, assieme alla sua derivata rispetto a $\rho$ in tutto il dominio $(D)$ e in ogni istante positivo. Noi considereremo, delle equazioni sopra scritte, solo soluzioni, che diremo regolari, cioè supporremo i valori di $v$ e $\rho$ (e di conseguenza quelli di $p$ ) funzioni continue, assieme alle loro derivate prime e seconde ${ }^{8}$ (rispetto alle

${ }^{6}$ Adopereremo il calcolo vettoriale di Burali-Forti e Marcolongo, esposto per essempio nel volumetto di P. BuRGatri "Calcolo vettoriale omografico", Hoepli, Milano, 1937. Comunque osserviamo che una omografia vettoriale generica $\alpha$ corrisponde a un tensore doppio, $K \alpha$ è la omografia corrispondente al tensore di $\alpha$ in cui sono scambiati gli indici, $D \alpha$ è la parte simmetrica del tensore che rappresenta $\alpha, I_{1}(\alpha)$ è l'invariante lineare dell'omografia $\alpha, d \mathbf{v} / d P$ l'omografia corrispondente al tensore derivato dal vettore $\mathbf{v},(d \mathbf{v} / d P) \mathbf{v}$ è il prodotto di composizione fra il tensore $d v / d P$ e il vettore $\mathbf{v}$, il gradiente di una omografia simmetrica è la divergenza del tensore corrispondente. Per la relazioni fra omografie e tensori si veda anche B. FinzI-M. PAStori "Calcolo tensoriale e applicazioni", Zanichelli, Bologna (1949), cap. III.

${ }^{7}$ Non ammetteremo la relazione di Stokes $3 \lambda+2 \mu=0$ del resto dubbia, (vedi C. TruesDELL-"The mechanical foundations of elasticity and fluid dynamics"-Journal of Rational Mechanics and Analysis I (1952) pp. 125-301) perche non necessaria per le nostre considerazioni.

${ }^{8}$ Le derivate seconde devono essere continue solo rispetto alle coordinate. Anzi questa ipotesi puo sostituirsi con l'altra, meno restrittiva, per cui il lavoro nel dominio $(D)$, dei termini dovuti alla viscosità, sia equivalente alla formula solita (di Lord RAYLEIGH) dell'energia dissipata. 
coordinate e al tempo) nel dominio quadrimensionale $(C)$ formato dall'insieme dei punti $P$, appartenenti al dominio $(D)$ in cui valgono quelle equazioni, e degli istanti $t$ compresi nell'intervallo $(0, T)$ dove $T$ è un valore finito del tempo, ma del resto grande quanto si vuole. Supporremo infine, conforme l'intuizione fisica, $\rho$ sempre positiva in $(C)$, quindi, per la sua continuità, esisterà un numero $m$ positivo tale che si possa supporre, in ogni caso:

$$
\rho \geqq m \text {. }
$$

Se il fluido è perfetto $(\lambda=\mu=0)$ le condizioni di regolarità si ridurranno a supporre $\mathrm{v}$ e $\rho$ continue in tutto $(C)$ (e $\rho$ soddisfacente alla (5)) con le derivate prime pure continue in $(C)$ ad eccezione, al più, di un numero finito di superfici di $(C)$ dove presentano discontinuità di prima specie; ciò per tener conto di eventuali moti ondosi (escluse ovviamente le onde di shock), comunque quelle derivate si ammetteranno sempre limitate in $(C)$. Possiamo perciò enunciare il seguente teorema di unicità: le equazioni (1), (2), (3), con la relazione (4), ammettono nel dominio $(D)$ e per ogni istante $t$ una sola soluzione regolare $\mathrm{v}, \rho$ qualora siano assegnati, per $t=0, i$ valori $d i \mathrm{v}$ e $\rho$ in tutto $(D)$ (condizioni iniziali); e se $(D) \grave{e}$ finito, siano assegnati per ogni $t>0$ i valori di $\mathrm{v}$ su tutta la superficie $\sigma$ che limita (D) e, nei punti di $\sigma$ dove $\mathbf{v} \cdot \mathbf{n}$ è negativo (n versore normale a $\sigma$ e diretto verso l'esterno $d i(D)$ ) cioè nei punti di $\sigma$ da cui entra il fluido, sia assegnato il valore di $\rho$ (condizioni al contorno). Se il fluido è perfetto, le condizioni al contorno possono anche ridursi ad assegnare su $\boldsymbol{\sigma}$ il valore $d i \mathbf{v} \cdot \mathbf{n} e$ che questo valore sia positivo o nullo, cioè basta supporre che il fluido non possa entrare in $(D)$. Se poi $(D)$ si estende all'infinito, occorrono alcune condizioni di convergenza all'infinito, ad esempio si può supporre la differenza fra $\rho \mathrm{e} \mathbf{\mathrm { v }} \boldsymbol{e}$ i valori assegnati di queste grandezze all'infinito ( $i$ valori di $\rho$ all'infinito si supporranno positivi onde valga (5)) infinitesima di ordine superiore a $\frac{3}{2}$ e, se il fluido è viscoso, le derivate della velocità (rispetto alle coordinate) infinitesime di ordine superiore $a \frac{1}{2}$.

3. Per dimostrare l'enunciato teorema di unicità, sostituiamo la (3) nella (1), sicchè le (1), (2), (3) equivalgono alle equazioni:

$$
\begin{aligned}
\rho \frac{\partial \mathbf{v}}{\partial t}+\rho \frac{d \mathbf{v}}{d P} \mathbf{v}= & -f^{\prime}(\rho) \operatorname{grad} \rho+\operatorname{grad} \beta+\mathbf{F}(\rho, P, t) \\
& \frac{\partial \rho}{\partial t}+\operatorname{div}(\rho \mathbf{v})=0 .
\end{aligned}
$$

Ora supponiamo, per assurdo, che le (6) e (7) abbiano due differenti soluzioni regolari, soddisfacenti però alle stesse condizioni iniziali e al contorno. Indichiamo con $\rho$ e $\mathbf{v}$ la prima di queste soluzioni, l'altra con $\rho+\rho_{1}, \mathbf{v}+\mathbf{v}_{1}$; se proveremo che $\rho_{1}$ e $\nabla_{1}$ sono identicamente nulle nel dominio $(D)$ e in ogni istante il nostro teorema di unicità sarà provato. Naturalmente $\rho+\rho_{1}, \mathbf{v}+\mathbf{v}_{1}$ soddisferanno alle stesse proprietà di $\rho$ e v. In particolare $\rho+\rho_{1}$ avrà un limite inferiore positivo che indicheremo ancora con $m$ cioè $\rho+\rho_{1}$ soddisferà la (5). 
Poichè $\rho+\rho_{1} \mathrm{e} \mathbf{v}+\mathbf{v}_{1}$ sono soluzioni di (6) e (7) avremo:

$$
\begin{gathered}
\left(\rho+\rho_{1}\right) \frac{\partial\left(\mathbf{v}+\mathbf{v}_{1}\right)}{\partial t}+\left(\rho+\rho_{1}\right) \frac{d\left(\mathbf{v}+\mathbf{v}_{1}\right)}{d P}\left(\mathbf{v}+\mathbf{v}_{1}\right) \\
=\mathrm{F}\left(\rho+\rho_{1}, P, t\right)-f^{\prime}\left(\rho+\rho_{1}\right) \operatorname{grad}\left(\rho+\rho_{1}\right)+\operatorname{grad}\left(\beta+\beta_{1}\right) \\
\frac{\partial\left(\rho+\rho_{1}\right)}{\partial t}+\operatorname{div}\left[\left(\rho+\rho_{1}\right)\left(\mathbf{v}+\mathbf{v}_{1}\right)\right]=0,
\end{gathered}
$$

con:

$$
\beta_{1}=2 \mu D \frac{d \nabla_{1}}{d P}+\lambda \operatorname{div} \nabla_{1} .
$$

Sottraendo (6) da (8), (7) da (9) ricaviamo9:

$$
\begin{gathered}
\rho_{1} \frac{\partial\left(\mathbf{v}+\mathbf{v}_{1}\right)}{\partial t}+\rho \frac{\partial \mathbf{v}_{1}}{\partial t}+\rho_{1} \frac{d\left(\mathbf{v}+\mathbf{v}_{1}\right)}{d P}\left(\mathbf{v}+\mathbf{v}_{1}\right)+\rho \frac{d \mathbf{v}_{1}}{d P}\left(\mathbf{v}+\mathbf{v}_{1}\right)+\rho \frac{d \mathbf{v}}{d P} \mathbf{v}_{1} \\
=\mathbf{F}\left(\rho+\rho_{1}\right)-\mathbf{F}(\rho)-f^{\prime}\left(\rho+\rho_{1}\right) \operatorname{grad} \rho_{1} \\
-\left[f^{\prime}\left(\rho+\rho_{1}\right)-f^{\prime}(\rho)\right] \operatorname{grad} \rho+\operatorname{grad} \beta_{1} \\
\frac{\partial \rho_{1}}{\partial t}+\operatorname{div}\left(\rho_{1} \mathbf{v}\right)+\operatorname{div}\left[\left(\rho+\rho_{1}\right) \mathbf{v}_{1}\right]=0 .
\end{gathered}
$$

4. Ciò posto, detto $V$ il volume del dominio $(D)$, consideriamo l'expressione:

$$
\begin{gathered}
A=-\int_{v} \rho_{1} \frac{\partial\left(\mathrm{v}+\mathrm{v}_{1}\right)}{\partial t} \cdot \mathrm{v}_{1} d V-\int_{V} \rho_{1} \frac{d\left(\mathrm{v}+\mathrm{v}_{1}\right)}{d P}\left(\mathrm{v}+\mathrm{v}_{1}\right) \cdot \mathrm{v}_{1} d V \\
-\int_{V} \rho \frac{d \mathbf{v}_{1}}{d P}\left(\mathrm{v}+\mathrm{v}_{1}\right) \cdot \mathrm{v}_{1} d V-\int_{V} \rho \frac{d \mathbf{v}}{d P} \mathbf{v}_{1} \cdot \mathrm{v}_{1} d V
\end{gathered}
$$

$$
\begin{aligned}
+\int_{V}\left[\mathbf{F}\left(\rho+\rho_{1}\right)-\mathbf{F}(\rho)\right] \cdot \nabla_{1} d V-\int_{\nabla} & {\left[f^{\prime}\left(\rho+\rho_{1}\right)-f(\rho)\right] \operatorname{grad} \rho \cdot \mathbf{v}_{1} d V } \\
& +\int_{\nabla} \operatorname{grad} \beta_{1} \cdot \mathrm{v}_{1} d V+\int_{V} \frac{\partial \rho}{\partial t} v_{1}^{2} d V .
\end{aligned}
$$

Avremo, indicando rispettivamente con $N_{1}$ e $N_{2}$ l'estremo superiore di $\left|\partial\left(\mathbf{v}+\mathbf{v}_{1}\right) / \partial t\right|$ e di $\left|d\left(\mathbf{v}+\mathbf{v}_{1}\right) / d P\left(\mathbf{v}+\mathbf{v}_{1}\right)\right|$ in $(C)$,

$$
\begin{aligned}
\left|-\int_{V} \rho_{1} \frac{\partial\left(\mathbf{v}+\mathbf{v}_{1}\right)}{\partial t} \cdot \mathrm{v}_{1} d V\right| \leqq N_{1} \int_{V} \rho_{1} v_{1} d V & \quad \leqq \frac{1}{2} N_{1}\left[\int_{V} \rho_{1}^{2} d V+\int_{V} v_{1}^{2} d V\right], \\
\left|-\int_{V} \rho_{1} \frac{d\left(\mathrm{v}+\mathrm{v}_{1}\right)}{d P}\left(\mathrm{v}+\mathrm{v}_{1}\right) \cdot \mathrm{v}_{1} d V\right| & \leqq N_{2} \int_{V} \rho_{1} v_{1} \leqq \frac{1}{2} N_{2}\left[\int_{V} \rho_{1}^{2} d V+\int_{V} v_{1}^{2} d V\right] ;
\end{aligned}
$$

${ }^{9}$ Scriveremo, per brevità, $\mathbf{F}\left(\rho+\rho_{1}\right)$ e $\mathbf{F}\left(\rho_{1}\right)$ in luogo di $\mathbf{F}\left(\rho+\rho_{1}, P, t\right), \mathbf{F}\left(\rho_{1}, P, t\right)$. 
e d'altra parte si ha:

$$
\begin{aligned}
-\int_{V} \rho \frac{d \nabla_{1}}{d P} & \left(\mathrm{v}+\mathrm{v}_{1}\right) \cdot \mathrm{v}_{1} d V \\
& =-\int_{V} \rho K \frac{d \mathrm{v}_{1}}{d P} \mathrm{v}_{1} \cdot\left(\mathrm{v}+\mathrm{v}_{1}\right) d V \\
& =-\int_{\nabla} \rho \operatorname{grad} \frac{1}{2} v_{1}^{2} \cdot\left(\mathrm{v}+\mathrm{v}_{1}\right) d V \\
& =-\int_{V} \operatorname{div}\left[\frac{1}{2} \mathrm{v}_{1}^{2} \rho\left(\mathrm{v}+\mathrm{v}_{1}\right)\right] d V+\frac{1}{2} \int_{V} v_{1}^{2} \operatorname{div}\left[\rho\left(\mathrm{v}+\mathrm{v}_{1}\right) d V\right. \\
& =-\frac{1}{2} \int_{\sigma} \rho v_{1}^{2}\left(\mathrm{v}+\mathrm{v}_{1}\right) \cdot \mathrm{n} d \sigma+\frac{1}{2} \int_{V} v_{1}^{2} \operatorname{div}\left[\rho\left(\mathrm{v}+\mathrm{v}_{1}\right)\right] d V .
\end{aligned}
$$

Ora, se il fluido è viscoso, il primo integrale all'ultimo membro di questa equazione è nullo perchè tale è $\mathbf{v}_{1}$, in quanto su $\sigma \mathrm{v}$ e $\mathbf{v}+\mathbf{v}_{1}$ devono assumere lo stesso valore. Se invece il fluido è perfetto e si fanno le ipotesi già esposte, $\left(\boldsymbol{\nabla}+\mathbf{v}_{1}\right) \cdot \mathbf{n} \geqq 0$ e quell'integrale è negativo. Quindi, detto $N_{3}$ l'estremo superiore $\operatorname{di}\left|\operatorname{div}\left[\rho\left(\boldsymbol{v}+\mathbf{v}_{1}\right)\right]\right|$ in (C) abbiamo:

(15) $-\int_{V} \frac{d \mathrm{v}_{1}}{d P}\left(\mathrm{v}+\mathrm{v}_{1}\right) \cdot \mathrm{v}_{1} d V \leqq \frac{1}{2}\left|\int_{V} v_{1}^{2} \operatorname{div}\left[\rho\left(\mathrm{v}+\mathrm{v}_{1}\right)\right] d V\right| \leqq \frac{1}{2} N_{3} \int_{V} v_{1}^{2} d V$.

Poiche le derivate di $\mathrm{v}$ sono limitate e $\rho$ è supposto limitato, esiste un numero $N_{4}$ tale che per ogni $P$ di $(D)$ e per ogni $t$ di $(0, T)$ si ha ${ }^{10}$ :

$$
\left|\rho \frac{d \mathrm{v}}{d P} \mathrm{v}_{1} \cdot \mathrm{v}_{1}\right| \leqq N_{4} v_{1}^{2} .
$$

Se poi $N_{5}$ è l'estremo superiore di $|\partial \rho / \partial t|$ in (C) vale la relazione:

$$
\left|\int_{V} \frac{\partial \rho}{\partial t} v_{1}^{2} d V\right| \leqq N_{5} \int_{V} v_{1}^{2} d V .
$$

Inoltre

$$
\begin{gathered}
\int_{V} \operatorname{grad} \beta_{1} \cdot \mathbf{v}_{1} d V=\int_{V} \operatorname{div}\left(\beta_{1} \mathbf{v}_{1}\right) d V-\int_{V} I_{1}\left(\beta_{1} D \frac{d \mathbf{v}_{1}}{d P}\right) d V \\
=\int_{\sigma} \beta_{1} \mathbf{v}_{1} \cdot \mathbf{n} d \sigma-\int_{V} I_{1}\left(\beta_{1} D \frac{d \mathbf{v}_{1}}{d P}\right) d V \\
=-\int_{V} I_{1}\left(\beta_{1} D \frac{d \mathbf{v}_{1}}{d P}\right) d V \leqq 0
\end{gathered}
$$

conforme all'ipotesi che si abbia energia dissipata.

Dette poi, $X, Y, Z$, le componenti sugli assi di F, e detto $N_{6}$ il massimo di

${ }^{10}$ Cfr. D. Graffi, Nota citata pag. 133. 
$|\partial X / \partial \rho|,|\partial Y / \partial \rho|,|\partial Z / \partial \rho|$ per $\rho$ compreso fra l'estremo superiore e l'estremo inferiore di $\rho$ e $\rho+\rho_{1}$ in $(C)$ e per $P$ e $t$ pure in $(C)$, si ha, dal teorema del valor medio:

$$
\begin{aligned}
\left|\left[\mathbf{F}\left(\rho+\rho_{1}\right)-\mathbf{F}(\rho)\right] \cdot \nabla_{1}\right| & \leqq N_{6}\left|\rho_{1} v_{1 x}\right|+N_{6}\left|\rho_{1} v_{1 y}\right|+N_{6}\left|\rho_{1} v_{1 z}\right| \\
& \leqq 2 N_{6} \rho_{1} v_{1} \leqq N_{6}\left({\rho_{1}}^{2}+v_{1}{ }^{2}\right) .
\end{aligned}
$$

Infine, detto $N^{\prime}$ il massimo di $\left|f^{\prime \prime}(\rho)\right|$ per $\rho$ variabile fra l'estremo inferiore e l'estremo superiore di $\rho$ e $\rho+\rho_{1}$ in $(C), N^{\prime \prime}$ l'estremo superiore di $|\operatorname{grad} \rho|$, detto infine $N_{7}$ il prodotto $N^{\prime} N^{\prime \prime}$ si ottiene:

$$
\left|\left[f^{\prime}\left(\rho+\rho_{1}\right)-f^{\prime}(\rho)\right] \operatorname{grad} \rho \cdot v_{1}\right| \leqq N_{7} \rho_{1} v_{1} \leqq \frac{1}{2} N_{7}\left(\rho_{1}{ }^{2}+v_{1}{ }^{2}\right) .
$$

Allora tenendo presente prima (18) e poi (13), (14), (15), (16), (17), (19) e (20) ricaviamo:

$$
\begin{aligned}
A \leqq\left[\frac{1}{2}\left(N_{1}+N_{2}+N_{3}+N_{7}\right)+\right. & \left.N_{4}+N_{5}+N_{6}\right] \int_{V} v_{1}^{2} d V \\
& +\left[\frac{1}{2}\left(N_{1}+N_{2}+N_{7}\right)+N_{6}\right] \int_{V} \rho_{1}^{2} d V .
\end{aligned}
$$

5. Consideriamo l'espressione:

$$
\begin{aligned}
f^{\prime}\left(\rho+\rho_{1}\right) \operatorname{grad} \rho_{1} \cdot \nabla_{1}= & f^{\prime}\left(\rho+\rho_{1}\right) \operatorname{div}\left(\rho_{1} \mathbf{v}_{1}\right)-f^{\prime}\left(\rho+\rho_{1}\right) \rho_{1} \operatorname{div} \mathbf{v}_{1} \\
= & \operatorname{div}\left[f^{\prime}\left(\rho+\rho_{1}\right) \rho_{1} \mathbf{v}_{1}\right]-\rho_{1} \mathbf{v}_{1} \cdot \operatorname{grad} f^{\prime}\left(\rho+\rho_{1}\right) \\
& -f^{\prime}\left(\rho+\rho_{1}\right) \rho_{1} \operatorname{div} \mathbf{v}_{1} .
\end{aligned}
$$

Ora da (11):

$$
\operatorname{div} \mathbf{v}_{1}=-\frac{1}{\rho+\rho_{1}}\left[\frac{\partial \rho_{1}}{\partial t}+\operatorname{div}\left(\rho_{1} \mathbf{v}\right)+\operatorname{grad}\left(\rho+\rho_{1}\right) \cdot \mathbf{v}_{1}\right]
$$

Sostituendo in (22):

$$
\begin{aligned}
f^{\prime}\left(\rho+\rho_{1}\right) \operatorname{grad} \rho_{1} \cdot \mathbf{v}_{1} & =\operatorname{div}\left[f^{\prime}\left(\rho+\rho_{1}\right) \rho_{1} \mathbf{v}_{1}\right]-\rho_{1} \mathbf{v}_{1} \cdot \operatorname{grad} f^{\prime}\left(\rho+\rho_{1}\right) \\
+\frac{f^{\prime}\left(\rho+\rho_{1}\right) \rho_{1}}{\rho+\rho_{1}} & \frac{\partial \rho_{1}}{\partial t}+\frac{f^{\prime}\left(\rho+\rho_{1}\right) \rho_{1}}{\rho+\rho_{1}} \operatorname{div}\left(\rho_{1} \mathbf{v}\right)+\frac{f^{\prime}\left(\rho+\rho_{1}\right)}{\rho+\rho_{1}} \operatorname{grad}\left(\rho+\rho_{1}\right) \cdot \mathbf{v}_{1} \rho_{1} \\
& =\operatorname{div}\left[f^{\prime}\left(\rho+\rho_{1}\right) \rho_{1} \mathbf{v}_{1}\right]+\frac{1}{2} \frac{f^{\prime}\left(\rho+\rho_{1}\right)}{\rho+\rho_{1}} \frac{\partial \rho_{1}^{2}}{\partial t} \\
& -\left[\operatorname{grad} f^{\prime}\left(\rho+\rho_{1}\right)-\frac{f^{\prime}\left(\rho+\rho_{1}\right)}{\rho+\rho_{1}} \operatorname{grad}\left(\rho+\rho_{1}\right)\right] \cdot \rho_{1} \mathbf{v}_{1} \\
& +\frac{f^{\prime}\left(\rho+\rho_{1}\right)}{\rho+\rho_{1}} \rho_{1}^{2} \operatorname{div} \mathbf{v}+\frac{f^{\prime}\left(\rho+\rho_{1}\right) \rho_{1}}{\rho+\rho_{1}} \operatorname{grad} \rho_{1} \cdot \mathbf{v} .
\end{aligned}
$$


D'altra parte si ha:

$$
\begin{aligned}
& \frac{f^{\prime}\left(\rho+\rho_{1}\right) \rho_{1}}{\rho+\rho_{1}} \operatorname{grad} \rho_{1} \cdot \mathbf{v}=\frac{1}{2} \frac{f^{\prime}\left(\rho+\rho_{1}\right)}{\rho+\rho_{1}} \operatorname{grad} \rho_{1}^{2} \cdot \mathbf{v} \\
& \quad=\frac{1}{2} \operatorname{grad}\left[\frac{f^{\prime}\left(\rho+\rho_{1}\right)}{\rho+\rho_{1}} \rho_{1}^{2}\right] \cdot \mathbf{v}-\frac{1}{2} \operatorname{grad} \frac{f^{\prime}\left(\rho+\rho_{1}\right)}{\rho+\rho_{1}} \cdot \rho_{1}^{2} \mathbf{v} \\
& \quad=\frac{1}{2} \operatorname{div} \frac{f^{\prime}\left(\rho+\rho_{1}\right) \rho_{1}^{2} \mathbf{v}}{\rho+\rho_{1}}-\frac{1}{2} \frac{f^{\prime}\left(\rho+\rho_{1}\right) \rho_{1}^{2}}{\rho+\rho_{1}} \operatorname{div} \mathbf{v}-\frac{1}{2} \operatorname{grad} \frac{f^{\prime}\left(\rho+\rho_{1}\right)}{\rho+\rho_{1}} \cdot \rho_{1}^{2} \mathbf{v} .
\end{aligned}
$$

Sostituendo in (22) si ottiene, dopo qualche altro passaggio:

$$
\begin{aligned}
f^{\prime}\left(\rho+\rho_{1}\right) \operatorname{grad} & \rho_{1} \cdot \mathbf{v}_{1}=\frac{1}{2} \frac{\partial}{\partial t} \frac{f^{\prime}\left(\rho+\rho_{1}\right) \rho_{1}^{2}}{\rho+\rho_{1}}-\frac{1}{2} \rho_{1}^{2} \frac{\partial}{\partial t} \frac{f^{\prime}\left(\rho+\rho_{1}\right)}{\rho+\rho_{1}} \\
& +\operatorname{div}\left[f^{\prime}\left(\rho+\rho_{1}\right) \rho_{1} \mathbf{v}_{1}\right]-\left[\operatorname{grad} f^{\prime}\left(\rho+\rho_{1}\right)\right. \\
& \left.-\frac{f^{\prime}\left(\rho+\rho_{1}\right)}{\rho+\rho_{1}} \operatorname{grad}\left(\rho+\rho_{1}\right)\right] \cdot \rho_{1} \mathbf{v}_{1}+\frac{1}{2} \frac{f^{\prime}\left(\rho+\rho_{1}\right)}{\rho+\rho_{1}} \rho_{1}^{2} \operatorname{div} \mathbf{v} \\
& +\frac{1}{2} \operatorname{div} \frac{f^{\prime}\left(\rho+\rho_{1}\right) \rho_{1}^{2} \mathbf{v}}{\rho+\rho_{1}}-\frac{1}{2} \operatorname{grad} \frac{f^{\prime}\left(\rho+\rho_{1}\right)}{\rho+\rho_{1}} \cdot \rho_{1}^{2} \mathbf{v} .
\end{aligned}
$$

Integriamo ora la (23) su tutto il volume $V$; indichiamo rispettivamente con $N_{8}$ e $N_{9}$ rispettivamente l'estremo superiore in $(C)$ di:

e di:

$$
\left|\operatorname{grad} f^{\prime}\left(\rho+\rho_{1}\right)-\frac{f^{\prime}\left(\rho+\rho_{1}\right)}{\rho+\rho_{1}} \operatorname{grad}\left(\rho+\rho_{1}\right)\right|
$$

$$
\left|-\frac{1}{2} \frac{\partial}{\partial t} \frac{f^{\prime}\left(\rho+\rho_{1}\right)}{\rho+\rho_{1}}+\frac{1}{2} \frac{f^{\prime}\left(\rho+\rho_{1}\right)}{\rho+\rho_{1}} \operatorname{div} \mathbf{v}-\frac{1}{2} \operatorname{grad} \frac{f^{\prime}\left(\rho+\rho_{1}\right)}{\rho+\rho_{1}} \cdot \mathrm{v}\right| .
$$

Ovviamente $N_{8}$ e $N_{9}$ sono quantità finite perche $\rho+\rho_{1}$ soddisfa la (5) e $f^{\prime}(\rho)$ è limitata. Otteniamo così:

$$
\begin{array}{r}
-\int_{V} f^{\prime}\left(\rho+\rho_{1}\right) \operatorname{grad} \rho_{1} \cdot \mathbf{v}_{1} d V \leqq-\frac{1}{2} \int_{V} \frac{\partial}{\partial t} \frac{f^{\prime}\left(\rho+\rho_{1}\right) \rho_{1}^{2}}{\rho+\rho_{1}} d V+\frac{1}{2} N_{8} \int_{V}\left(\rho_{1}^{2}+v_{1}^{2}\right) d V \\
+N_{9} \int_{V} \rho_{1}^{2} d V-\int_{\sigma} f^{\prime}\left(\rho+\rho_{1}\right) \rho_{1} \mathbf{v}_{1} \cdot \mathbf{n} d \sigma-\frac{1}{2} \int_{\sigma} \frac{f^{\prime}\left(\rho+\rho_{1}\right) \rho_{1}^{2}}{\rho+\rho_{1}} \mathbf{v} \cdot \mathbf{n} d \sigma .
\end{array}
$$

Ora, poichè su $\sigma \mathbf{v}_{\mathbf{1}} \cdot \mathbf{n}$ è nulla, il penultimo integrale è nullo; poichè nei punti di $\sigma$ dove $\mathbf{v} \cdot \mathbf{n}$ è negativo $\rho_{1}$ è nulla, mentre, dove $\mathbf{v} \cdot \mathbf{n} \geqq 0$ il termine $f^{\prime}\left(\rho+\rho_{1}\right) \rho_{1}^{2} /$ $\left(\rho+\rho_{1}\right)$ è positivo, si ha che quell'integrale è positivo o nullo. Possiamo perciò concludere:

$$
\begin{aligned}
-\int_{V} f^{\prime}\left(\rho+\rho_{1}\right) \operatorname{grad} \rho_{1} \cdot \nabla_{1} d V \leqq & -\frac{1}{2} \int_{V} \frac{\partial}{\partial t} \frac{f^{\prime}\left(\rho+\rho_{1}\right) \rho_{1}^{2}}{\rho+\rho_{1}} d V \\
& +\frac{1}{2} N_{8} \int_{V} v_{1}^{2} d V+\left(\frac{1}{2} N_{8}+N_{9}\right) \int_{V} \rho_{1}^{2} d V .
\end{aligned}
$$


6. Ciò posto, integriamo la (10) dopo averla moltiplicata scalarmente per $\mathbf{v}_{\mathbf{1}}$, su tutto il volume $V$, otteniamo:

$$
\frac{1}{2} \int_{V} \frac{\partial\left(\rho v_{1}^{2}\right)}{\partial t} d V=A-\int_{V} f^{\prime}\left(\rho+\rho_{1}\right) \operatorname{grad} \rho_{1} \cdot v_{1} d V
$$

e ricordando (24) e (21), indicando con $M_{1}$ e $M_{2}$ la somma dei coefficienti (positivi) che moltiplicano gli integrali di $v_{1}^{2}$ e $\rho_{1}^{2}$ otteniamo:

$$
\frac{1}{2} \int_{V} \frac{\partial}{\partial t}\left(\rho v_{1}^{2}\right) d V+\frac{1}{2} \int_{V} \frac{\partial}{\partial t} \frac{f^{\prime}\left(\rho+\rho_{1}\right) \rho_{1}^{2}}{\rho+\rho_{1}} d V \leqq M_{1} \int_{V} v_{1}^{2} d V+M_{2} \int_{V} \rho_{1}^{2} d V
$$

Ora integrando questa relazione da 0 a $t$, tenendo presente che $\rho_{1}$ e $v_{1}$ sono nulle per $t=0$ si ha:

$$
\frac{1}{2} \int_{V} \rho v_{1}^{2} d V+\frac{1}{2} \int_{V} \frac{f^{\prime}\left(\rho+\rho_{1}\right) \rho_{1}^{2}}{\rho+\rho_{1}} d V \leqq \int_{0}^{t} M_{1} d t \int_{V} v_{1}^{2} d V+\int_{0}^{t} M_{2} d t \int_{V} \rho_{1}^{2} d V .
$$

Ma $\rho \geqq m$ e $f^{\prime}\left(\rho+\rho_{1}\right) /\left(\rho+\rho_{1}\right)$ è funzione continua positiva in tutto $(C)$, quindi detto $l(l>0)$ il suo estremo inferiore in questo dominio:

$$
\frac{1}{2} m \int_{V} v_{1}^{2} d V+\frac{1}{2} l \int_{V} \rho_{1}^{2} d V \leqq M_{1} \int_{0}^{t} d t \int_{V} v_{1}^{2} d V+M_{2} \int_{0}^{t} d t \int_{V} \rho_{1}^{2} d V .
$$

Ora indichiamo con $n$ il più piccolo fra $\frac{1}{2} m$ e $\frac{1}{2} l$, con $Q$ il più grande fra $M_{1}$ ed $M_{2}$, con $M$ il rapporto $Q / n$ abbiamo:

$$
\int_{V}\left(v_{1}^{2}+\rho_{1}^{2}\right) d V \leqq M \int_{0}^{t} d t \int_{V}\left(v_{1}^{2}+\rho_{1}^{2}\right) d V
$$

e da questa relazione si trae subito ${ }^{11}$, per ogni $t<T$ anzi, essendo $T$ arbitrario, per ogni $t$ :

$$
\int_{V}\left(v_{1}^{2}+\rho_{1}^{2}\right) d V=0
$$

da cui risulta subito che $\mathrm{v}_{1}$ e $\rho_{1}$ sono identicamente nulle per ogni $t$ in tutto $(D)$. Il teorema di unicità è perciò completamente provato.

11 Vedi D. Graffr, Nota citata, pag. 135. 